\title{
Assessing Vascularity of Osseous Spinal Metastases with Dual-Energy CT-DSA: A Pilot Study Compared with Catheter Angiography
}

\author{
(D) Y.-C. Huang, (DF.-Y. Tsuang, (D) C.-W. Lee, DC.-Y. Wu, and DY.-H. Lin
}

\begin{abstract}
BACKGROUND AND PURPOSE: Spine debulking surgery in patients with hypervascular spinal metastasis is associated with massive intraoperative blood loss, but currently, the vascularity of tumor is determined by invasive conventional angiography or dynamic contrast MR imaging. We aimed to investigate the usefulness of noninvasive dual-energy CT-DSA, comparing it with conventional angiography in evaluating the vascularity of spinal metastasis.
\end{abstract}

MATERIALS AND METHODS: We conducted a retrospective study from January to December 2018. A total of 15 patients with spinal metastasis undergoing dual-energy CT, conventional DSA, and subsequent debulking surgery were included. CT-DSA images were produced after rigid-body registration and subtraction between CT phases. Qualitative and quantitative assessments of tumor vascularity were conducted. Correlations between CT-DSA and conventional DSA results were evaluated using the Spearman coefficient. The mean enhancement in the estimated tumor volume and surgical blood loss was compared between hypervascular and nonhypervascular groups using the Wilcoxon rank sum test.

RESULTS: The CT-DSA and DSA results were strongly correlated, with $\rho=0.87(P<.001)$. The DSA and the quantitative enhancement index also showed a strong correlation with $\rho=0.83(P<.001)$. Wilcoxon rank sum testing between hypervascular and nonhypervascular CT-DSA groups showed a difference in enhancement indices $(P=.0003)$. The blood loss between the hypervascular and nonhypervascular groups was nonsignificant $(P=.09)$.

CONCLUSIONS: Dual-energy CT-DSA correlates well with conventional DSA in assessing the vascularity of spinal metastasis. It may serve as a noninvasive preoperative evaluation option before debulking surgery.

ABBREVIATION: DECT $=$ dual-energy $C T$

B ony metastases of the spine can be very detrimental to a patient's quality of life because they can cause severe pain, instability, and neurologic deficits secondary to spinal cord and root compression. ${ }^{1,2}$ In selected cases, debulking surgery improves local control and quality of life to a greater degree than conventional methods. ${ }^{3}$ However, aggressive spinal surgery could be complicated by massive intraoperative hemorrhage in hypervascular osseous metastases. Related studies have demonstrated that preoperative transarterial embolization might reduce intraoperative blood loss, ${ }^{4-9}$ especially for pathologies prone to hypervascular

Received November 13, 2018; accepted after revision March 6, 2019

From the Department of Medical Imaging (Y.-C.H., C.-W.L., Y.-H.L.), Division of Neurosurgery (F.-Y.T.), Department of Surgery, and Department of Anesthesiology (C.-Y.W.), National Taiwan University Hospital, Taipei, Taiwan.

Please address correspondence to Yen-Heng Lin, MD, MS, Department of Medical Imaging, National Taiwan University Hospital, No. 7, Chung-Shan South Rd, Taipei 100, Taiwan, ROC; e-mail: cryhungry@gmail.com

http://dx.doi.org/10.3174/ajnr.A6023 metastases such as thyroid carcinoma, renal cell carcinoma, and hepatocellular carcinoma. However, not all metastases from such tumors are hypervascular, ${ }^{5,10}$ and metastases from other tumor pathologies could be hypervascular as well. Therefore, it is recommended that tumor vascularity be assessed before spinal surgery. ${ }^{4}$ DSA is considered a reference standard for vascularity assessment of bone tumors. However, DSA is an invasive imaging tool, and quantitative measurements using DSA have not been comprehensively explored, to our knowledge.

$\mathrm{CT}$ is one of the most commonly available imaging tools in modern medicine. It is excellent at demonstrating osseous integrity and fracture margins at rapid scanning speeds. ${ }^{11}$ In cases of osseous metastases, the degree of enhancement is often hindered by the inherent high density of mineral components. Additionally, in small osseous metastases without substantial bone morphologic changes, conventional CT has poor detection abilities compared with MR imaging and PET. ${ }^{12,13}$ Therefore, CT plays a supplementary role in diagnosing spinal metastases. Recent ad- 
vancements in CT registration and subtraction have enabled demonstration of hypervascular bone tumors, marrow edema, and spinal arteries. ${ }^{14-16}$ The contrast-to-noise ratio can be optimized when the images are reconstructed at selected monoenergetic levels by performing dual-energy CT (DECT). ${ }^{17}$

In this pilot study, we evaluated the potential of dual-energy CT-DSA, compared with DSA, for assessing the vascularity of osseous spinal metastasis before debulking surgery.

\section{MATERIALS AND METHODS \\ Patients}

This study was approved by the institutional review board of our hospital. All patients who underwent dual-energy spinal CT angiography for osseous metastases from January to December 2018 at a single tertiary referral center were retrospectively reviewed. Patients were included if they underwent spinal surgery and DSA at the target levels with histopathology-proved metastatic malignancy. Patients were excluded from the analysis if they had prior spinal surgery at the target levels or if the image quality of DSA and CT was poor for post-image processing.

\section{CTA Acquisition and Image Postprocessing}

All CT scans were performed on a 256 -detector row fast-kVswitching DECT system (Revolution CT; GE Healthcare, Milwaukee, Wisconsin). The scan range contained at least 5 vertebral body levels centered at the target level. The scan range was extended in sacral and cervical lesions to include possible distal collateral blood supplies. The scans were obtained in dual-energy mode on a fast-kV-switching DECT system that switched tube potentials between 140 and $80 \mathrm{kV}$. Radiation doses were adjusted according to the diagnostic reference levels ${ }^{18,19}$ and previously reported doses that could identify the anterior spinal artery. ${ }^{20-22}$ Narrow collimation (between 4 and $8 \mathrm{~cm}$ ) and a low scan pitch factor (lower than 1) were used to reduce the conebeam artifacts and enhance the $\mathrm{z}$-axis resolution while maintaining a scan time of $<8$ seconds. We obtained 4 CT phases: noncontrast, early arterial, late arterial, and venous. Intravenous nonionic contrast medium was injected into the antecubital vein at a flow rate of 4 $\mathrm{mL} / \mathrm{s}$; the total contrast amount was between 60 and $80 \mathrm{~mL}$, which had been tailored to the scan time. Autoscan triggering was used with the ROI situated at the aorta at the starting position of the scan range. The scan started 2.6 seconds after the ROI had reached $100 \mathrm{HU}$ to acquire the early arterial phase; the late arterial phase started 4 seconds after the early arterial phase had been completed. The venous phase was then performed after a 60 -second delay. The patients were instructed to maintain shallow, slow breathing during the scans. The images were reconstructed on 0.625 -mm-thick contiguous slices with a 16- to 20-cm FOV.

A stand-alone workstation (Advantage Workstation, Version 4.7; GE Healthcare) was used for postprocessing images. First, a 40-keV monochromatic CT image was reconstructed from the DECT data file to enhance the contrast conspicuity. Late arterial phase images were then registered to a noncontrast scan through rigid-body registrations. Subtraction images between the late arterial phase and noncontrast phase images were then obtained to produce pure enhanced images, namely CT-DSA images. Figure 1 depicts CT-DSA before and after registration.

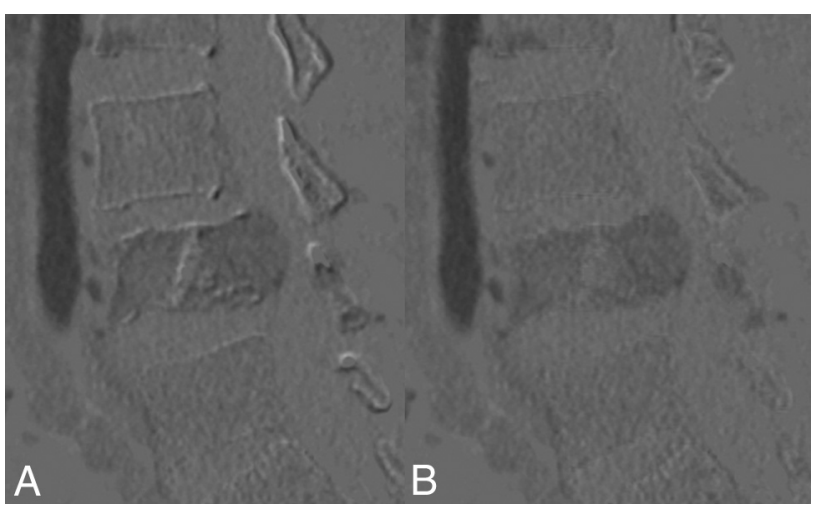

FIG 1. Sagittal subtraction CT before and after rigid-body registration. If we set the subtracted image to a proper window, the quality of registration can be assessed using a DSA-like visual inspection method. $A$, Unregistered image shows an overt misalignment artifact over bony edges. $B$, After solid-body registration, the number of artifacts is greatly reduced.

\section{Quantitative Measurement and Qualitative Evaluation of CT-DSA}

After the production of subtracted images, quantitative measurements were performed on a stand-alone workstation by one of the authors (with 4 years' experience in neuroradiology) blinded to the clinical information. The tumor mass was segmented along the axial plane, slice by slice, to form a 3D ROI. Vessels and misregistered artifacts were excluded from the selection. Tumor volume and mean enhancement were obtained from the resultant ROI. The following enhancement index was formulated to represent the percentage of tumor enhancement compared with that of the artery:

\section{Enhancement Index $=$}

Mean Enhancement of the Estimated Tumor Volume Enhancement of Aorta or Great Artery

For qualitative evaluation, the subtracted images were reformatted to standard 3-mm axial, coronal, and sagittal images with color-coded displays. The color scale was set between the part of the scan range that showed the best enhancement (typically the aorta) and zero Hounsfield units. The color map image was read on a standard PACS for clinical diagnostic purposes. Two neuroradiologists (with 9 and 4 years' experience, respectively) read the studies independently; they were blinded to the clinical information and catheter spinal angiography results. Tumor vascularity was classified into 5 grades: $0=$ enhancement lower than that of the nondiseased adjacent vertebral bodies, 1 = peak enhancement area similar to that of the nondiseased adjacent vertebral bodies, $2=$ peak enhancement area higher than that of the nondiseased adjacent vertebral bodies but lower than that of one-third of the aortic enhancement, 3 = peak enhancement area higher than that of one-third of the aortic enhancement, and $4=$ peak enhancement area higher than that of one-third of the aortic enhancement with the presence of early venous enhancement. Figure 2 shows the color map of CT-DSA alongside the corresponding catheter spinal angiography images.

AJNR Am J Neuroradiol 40:920-25 May 2019 www.ajnr.org 921 


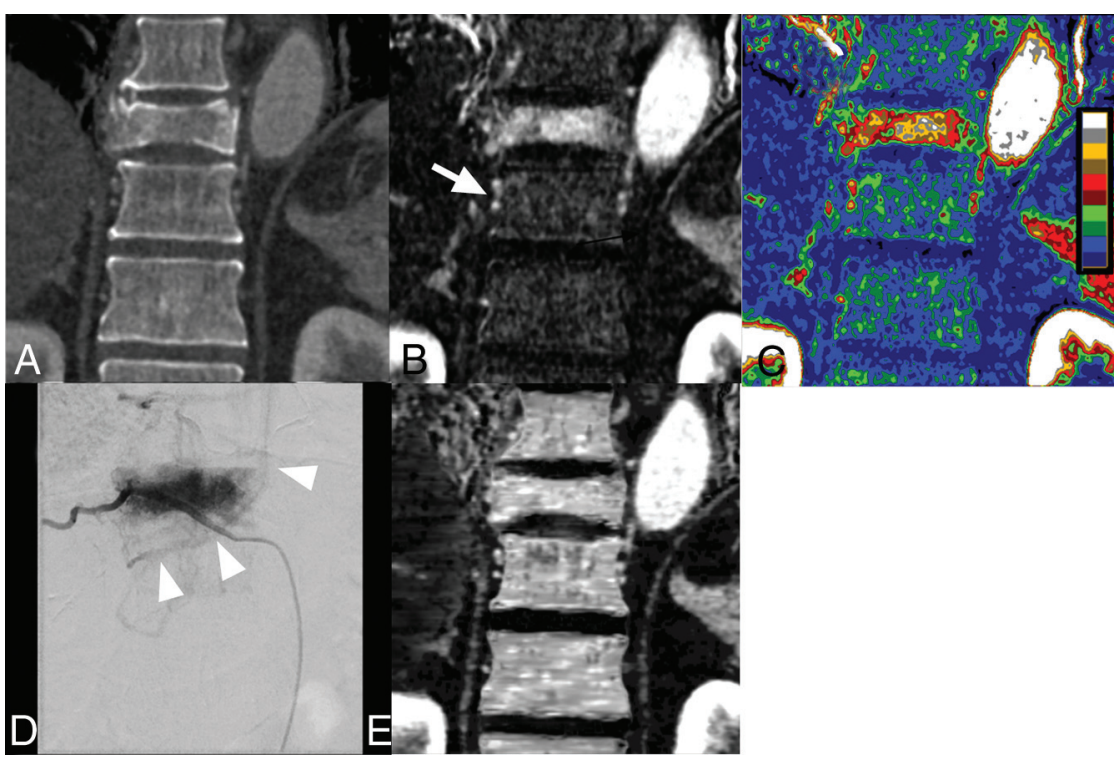

FIG 2. A 68-year-old male patient with hepatocellular carcinoma with a hypervascular bony metastasis in the T10 vertebral body. A, Coronal $40-\mathrm{keV}$ monoenergetic image of the late arterial phase shows a T10 vertebral body osteolytic lesion with a pathologic compression fracture. $B$, Coronal 40-keV subtracted CTA after registration shows that the T10 vertebral body had been replaced by a metastatic tumor with avid enhancement stronger than that of the marrow in the adjacent vertebral body. An earlier segmental draining vein (white arrow) parallel to the segmental artery is shown for comparison with a single enhancing segmental artery at the adjacent normal level. C, Color-coded display of the subtracted images demonstrates the degree of enhancement more clearly. The color map is set to a 10-stepped scale with $10 \%$ increments for each step to assist in visual grading. D, Frontal view of DSA at the same level shows a typical hypervascular bone metastasis. Early venous drainage to a hemizygous vein is noted (arrowheads), indicating arteriovenous shunting within the metastatic lesion. A smaller part of the vertebral body supplied by the left segmental artery is not opacified in this DSA image. $E$, lodine map derived from the material decomposition algorithm shows falsely high iodine content in normal bone marrow. The map failed to demonstrate the expected enhancement difference between the tumor and normal bone marrow because of limitations of the dual-energy CT iodine-based material decomposition in bony tissue.

\section{Spinal Angiography}

Catheter spinal angiography was performed and read by a neurointerventionalist (with 4 years' experience in neurointervention and 9 years' experience in neuroradiology) with the patient under local anesthesia. If a target metastasis was in the cervical spine, the cervical spinal angiography included bilateral vertebral angiography. For thoracolumbar disease, segmental artery angiography was performed at both the target and adjacent levels. For a target level lower than L3, iliac artery angiography was also performed. Tumor vascularity was classified into 5 categories according to the literature $^{23}: 0=$ decreased enhancement compared with that in a normal nondiseased vertebral body, $1=$ same degree of enhancement compared with that in a normal nondiseased vertebral body, $2=$ mild tumor blush, $3=$ moderate tumor blush with an opacity similar to that of the segmental artery proper, and $4=$ marked tumor blush with early arteriovenous shunting. The angiography study was also used to identify the Adamkiewicz and anterior spinal arteries.

\section{Preoperative Embolization}

Preoperative embolization was performed after the complete angiography study if no anterior spinal arteries had been identified at the target levels. For tumors in the thoracic-to-sacral regions, embolization was performed with particles (Contour and Embozene particles; Boston Scientific, Natick, Massachu-

\section{Statistical Analysis}

\section{RESULTS}

setts) at the target level and adjacent segmental arteries when tumor stain was present. The embolization was assisted by a coil for flow redistribution and proximal occlusion when necessary. ${ }^{24,25}$ For tumors at the cervical level and when the vertebral artery was encased by a tumor, coil embolization of the affected vertebral artery at both the distal and proximal tumor edges was performed when the collateral supply from the contralateral vertebral artery was adequate according to CTA and selective angiography.

\section{Surgical Procedure}

A surgical plan was developed by a multidisciplinary spine oncology board, and the treatment options were determined according to the Tomita survival scores, ${ }^{26}$ revised Tokuhashi Scores, ${ }^{27}$ and an algorithm proposed by Cappuccio and Boriania. ${ }^{28}$ All patients underwent intralesional debulking surgery or total en bloc spondylectomy, in which surgeons removed the vertebrae with the tumor in piecemeal fashion or as a whole. Vertebral reconstructions were performed 2 levels above and below the resected vertebra with prefabricated metallic implants or polymethyl methacrylate and posterior fixation.

Individual patient data were provided in detail. Spearman rank correlation of DSA to the enhancement index and CT-DSA grade was conducted. The interrater reliability of qualitative data was evaluated by weighted $\kappa$ values. We further dichotomized the patients into hypervascular and nonhypervascular groups according to their CT-DSA enhancement grades. Mean enhancement in the estimated tumor volume and surgical blood loss was compared between the 2 groups using the Wilcoxon rank sum test. The significance level was set at .05. All statistical analyses were performed using SAS software, Version 9.4 (SAS Institute, Cary, North Carolina).

A total of 47 patients evaluated for preoperative spinal CTA were analyzed during the study period. Seven patients did not undergo surgery after the multidisciplinary board judged that surgery was unnecessary, 17 patients had no preoperative DSA, 2 patients had pathologies other than metastases, and 6 patients were excluded from this study because of prior palliative surgery. Finally, 15 patients were included for analysis. Among them, 3 patients had 2 target levels, and the remaining 12 patients had 1 target level. The tumor types varied, comprising 1 melanoma, 1 colonic carcinoma, 1 renal cell carcinoma, 1 thyroid cancer, 1 parotid epithelial-myoepithelial carcinoma, 3 breast cancers, 1 cervical cancer, 2 


\begin{tabular}{|c|c|c|c|c|c|c|c|c|c|c|c|}
\hline Patient & Sex & $\begin{array}{l}\text { Age } \\
\text { (yr) }\end{array}$ & Tumor Type & $\begin{array}{l}\text { Tumor } \\
\text { Location }\end{array}$ & $\begin{array}{c}\text { DSA } \\
\text { Grade, } \\
\text { Reader } \\
1 / 2\end{array}$ & $\begin{array}{c}\text { CT-DSA } \\
\text { Grade, } \\
\text { Reader } \\
1 / 2\end{array}$ & $\begin{array}{c}\text { Estimated } \\
\text { Tumor } \\
\text { Volume } \\
\left(\mathrm{cm}^{3}\right)\end{array}$ & $\begin{array}{l}\text { Enhancement } \\
\text { Index }\end{array}$ & $\begin{array}{c}\text { Surgical } \\
\text { Approach/Procedure }\end{array}$ & $\begin{array}{l}\text { Preoperative } \\
\text { Embolization }\end{array}$ & $\begin{array}{l}\text { Blood } \\
\text { Loss } \\
\left(\mathrm{cm}^{3}\right)\end{array}$ \\
\hline 1 & $M$ & 67 & Melanoma & L3 & $1 / 1$ & $1 / 1$ & 78.83 & 0.1574 & Posterior/debulking & Yes & 2300 \\
\hline 2 & M & 55 & Colon cancer & L4 & $3 / 3$ & $3 / 3$ & 49.59 & 0.1659 & Posterior/debulking & Yes & 3950 \\
\hline 3 & $\mathrm{~F}$ & 44 & Renal cell carcinoma & $\mathrm{C} 4$ & $4 / 4$ & $3 / 4$ & 16.28 & 0.6254 & Anterior/debulking & Yes & 600 \\
\hline 4 & M & 72 & Thyroid cancer & $\mathrm{C} 7$ & $2 / 1$ & $2 / 3$ & 11.93 & 0.1522 & Anterior/debulking & Yes & 800 \\
\hline 5 & M & 54 & Breast cancer & L4 & $3 / 3$ & $4 / 4$ & 36.74 & 0.2586 & Posterior/debulking & Yes & 10,000 \\
\hline 6 & M & 69 & Hepatocellular carcinoma & T10 & $4 / 4$ & $3 / 4$ & 18.14 & 0.2917 & Posterior/debulking & Yes & 4600 \\
\hline 7 & $\mathrm{~F}$ & 69 & Lung cancer & $\mathrm{T} 4$ & $1 / 0$ & $1 / 1$ & 10.86 & 0.1653 & Posterior/debulking & Yes & 3000 \\
\hline 8 & M & 74 & Hepatocellular carcinoma & Tा0 & $2 / 3$ & $1 / 1$ & 22.25 & 0.0673 & Posterior/debulking & No & 1000 \\
\hline 9 & $\mathrm{~F}$ & 58 & Cervical cancer & L4-5 & $2 / 4$ & $2 / 2$ & 91.14 & 0.0734 & Posterior/debulking & Yes & 1400 \\
\hline 10 & $\mathrm{~F}$ & 51 & Lung cancer & L4-5 & $4 / 3$ & $3 / 3$ & 11.61 & 0.2183 & Posterior/debulking & Yes & 3400 \\
\hline 11 & M & 60 & Hepatocellular carcinoma & T4 & $4 / 4$ & $4 / 4$ & 14.19 & 0.3325 & Posterior/debulking & Yes & 1400 \\
\hline 12 & M & 64 & Hepatocellular carcinoma & $\mathrm{T} 3$ & $4 / 4$ & $4 / 3$ & 26.47 & 0.1888 & Posterior/debulking & Yes & 2000 \\
\hline 13 & $\mathrm{~F}$ & 64 & Breast cancer & T3-4 & $2 / 2$ & $3 / 2$ & 29.06 & 0.1107 & Posterior/debulking & Yes & 600 \\
\hline 14 & $\mathrm{~F}$ & 44 & Breast cancer & L5 & $0 / 1$ & $2 / 2$ & 42.86 & 0.0450 & Posterior/debulking & Yes & 200 \\
\hline 15 & $\mathrm{~F}$ & 58 & $\begin{array}{l}\text { Parotid epithelial- } \\
\text { myoepithelial } \\
\text { carcinoma }\end{array}$ & $\mathrm{T} 4$ & $1 / 2$ & $2 / 2$ & 4.05 & 0.0339 & Posterior/en bloc & No & 2700 \\
\hline
\end{tabular}

lung cancers, and 4 hepatocellular carcinomas. The target vertebrae included 2 cervical, 7 thoracic, and 6 lumbar vertebrae. Thirteen patients underwent preoperative embolization before surgery. All patients underwent debulking surgery for spinal metastases; among them, 2 patients had anterior approaches for cervical metastasis debulking, and 13 with thoracolumbar metastases underwent debulking surgery through a single posterior approach. Individual patient data are listed in the Table.

The volume CT dose index of the present study was 9.33-20.93 mGy (median, $12.64 \mathrm{mGy}$ ) for a single late CTA phase and 5.98$20.93 \mathrm{mGy}$ (median, $12.25 \mathrm{mGy}$ ) for other single phases.

Spearman coefficients demonstrated a strong correlation between the DSA and CT-DSA grades, with $\rho=0.87(P<.001)$. The DSA grade and CT-DSA enhancement index were also strongly correlated, with $\rho=0.83(P<.001)$. For the interrater reliability of DSA and CT-DSA, the weighted $\kappa$ values were 0.64 (range, $0.43-0.84)$ and 0.74 (95\% CI, 0.51-0.96), respectively. The patients with CT-DSA grades 3 and 4 were assigned to the hypervascular group, and those with grades 0,1 , and 2 were assigned to the nonhypervascular group. The Wilcoxon rank sum test revealed a difference in the enhancement indices of the 2 groups $(P=.0003)$. The hypervascular group had higher mean surgical blood loss than the nonhypervascular group $(3707 \pm 3121$ versus $1500 \pm$ $1041 \mathrm{~mL})$; however, this difference was not significant $(P=.09)$.

\section{DISCUSSION}

Spine bony metastases often result in severe pain, instability, and neurologic deficits ${ }^{1,2}$ in patients with late-stage malignancies. Debulking surgeries in selected cases provide improved local control rates and neurologic outcomes but can be complicated by massive intraoperative blood loss. ${ }^{3}$ Currently, DSA is used as a reference standard for evaluating tumor vascularity before surgery. ${ }^{10,23,29}$ However, DSA is an invasive procedure that has only moderate interrater and intrarater reliability for classifying the vascularity of spinal metastases. ${ }^{30}$ In our study, the CT-DSA grading system and quantification measurements were strongly correlated with the DSA grading system.

DSA grading can be suboptimal because of variations in angiography techniques, anatomy, and tumor burden. In case 5 of our series, DSA exhibited lower vascularity than CT-DSA (grade 3 versus 4) because the planned injection rate was insufficient for the hypertrophied artery. Conversely, the degree of perfused arterial enhancement was uniform in the CTA image, which was unaffected by technique or anatomy variation during catheterization. Comparisons between adjacent levels may be more reliable.

Currently, there is no consensus on the CT classification of tumor vascularity. The proposed CT-DSA classification criteria were designed to be clinically useful and similar to the DSA classification criteria. On the basis of discussions with spinal surgeons of the multidisciplinary spine oncology board, we stratified grades 3 and 4 and 0,1 , and 2 as the closest matches for clinical experience. The development of an appropriate and clinically relevant classification scheme requires well-designed studies on a large scale with a sufficient follow-up duration. For a pilot study with a low case number, a solid suggestion regarding classification criteria is difficult to postulate. However, we believe that our current study demonstrates that CT-DSA is practical and potentially useful as a robust noninvasive tool for the preoperative evaluation of tumor vascularity.

In related studies, specialized MR imaging protocols applying dynamic contrast enhancement and high-resolution MRA have been described as a means of obtaining information regarding perfusion and arterial anatomy; these protocols are complex and require specialized MR imaging sequences that are not routinely performed. ${ }^{23,29,31}$ Meanwhile, conventional MR imaging sequences might not provide sufficient information regarding the anatomy or degree of vascularity. ${ }^{31}$ The CTA protocol described in our study requires only minimal modification from standard CTA protocols for other body parts; thus, this protocol might be easier to incorporate into clinical practice. The correlation between CT-DSA vascularity grading and intraoperative blood loss was nonsignificant in this study. However, there is a trend suggesting that high-grade tumors have high intraoperative blood loss. Because only 15 patients were included in this study, the primary reason for the nonsignificant result $(P=.09)$ was insufficient statistical power and other uncontrolled parameters, including tumor location, tumor size, surgical method, and degree of embolization. Thus, in this study, conclusions were difficult to draw, and further large-scale studies are needed to clarify the correlation between CT-DSA and intraoperative blood loss.

DECT scans are mainly used to optimize the contrast-to-noise 
ratios and improve the conspicuity of the anterior spinal and Adamkiewicz arteries. ${ }^{32}$ In this study, the unique abilities of DECT to perform material decomposition and virtual noncontrast studies were not comprehensively explored for a reason. We initially attempted to use a material decomposition algorithm to produce iodine mapping; however, material decomposition algorithms operate poorly in vertebral body scans. Iodine maps produced by commercial standard material decomposition algorithms (with 2 base material pairs: iodine-hydroxyapatite and iodine-water) and custom-made software that implements multiple material decomposition algorithms, as described elsewhere, ${ }^{33}$ show falsely high iodine content in normal bone marrow (Fig $2 E$ ). In the present study, 11 of the 15 cases (73\%) exhibited higher iodine content in normal vertebral marrow than did tumors on iodine maps derived from multiple material decomposition algorithms. We believe that this finding was a result of the implemented algorithm being unable to decompose 4 materials simultaneously. ${ }^{34-37}$ In addition to the 3-material model used in the literature for demonstrating bone marrow content distributions, ${ }^{38,39}$ iodine is added as a fourth material in enhanced spine studies. The introduction of a fourth material violates the assumption of the 3-material decomposition algorithm and renders the algorithm unsuitable for use in contrast spine CT. Virtual unenhanced CT images have the same limitations because they are based on the algorithm that performs multiple material decompositions. Nevertheless, the role of DECT in vertebral metastases is yet to be determined, and we still consider DECT an appealing imaging tool for vertebral body analysis worthy of further investigation.

In the present study, CT-DSA images were produced with precontrast and late CTA scans only. The early arterial phases are not used in CT-DSA, but it depicted segmental arteries well, because the late arterial phase might have fading enhancements in arteries that preclude small artery anatomic assessment. The venous phase provides an enhanced series to make differential diagnoses and can depict adjacent large venous structures (eg, the azygos vein and the epidural venous plexus). These structures may not be identified during an operation because of the limited surgical FOV.

Although CT-DSA was performed with DECT scans in our study, registration and subtraction can be performed without the dual-energy scan mode. We believe that DECT scans are not mandatory for subtracting CT to evaluate vascularity, and CT-DSA can be used at most CT sites.

Our study had several limitations. The first limitation was the small size of the pilot study group, which prevented further control and analysis of variables. A study with a larger sample size is warranted to confirm the correlations with general patient conditions. The second limitation concerns the retrospective nature of this study. The subjects analyzed in this study were patients who had undergone surgical interventions, which only represent a subgroup of all patients. This feature rendered the analysis vulnerable to selection bias. The third limitation was the use of registration and subtraction, which may not be available at every CT site. However, solid body registration and subtraction are fast and relatively easy postprocessing steps that are commercially available from most CT vendors and in open-source image-processing packages. ${ }^{40}$ For CT sites where registration is not available, subtraction of unregistered phases might be helpful.

The final limitation was the high radiation dose in the proposed protocol. Although we adjusted our radiation exposure levels in accordance with reported diagnostic reference levels in the literature and implemented standard dose-reduction techniques, radiation exposure remained high because of multiple phase acquisitions and a high single-phase radiation dose for spine CTs. Careful patient selection is warranted when using this protocol in clinics. Patients with malignant osseous spinal metastases are more likely to benefit from preoperative vascular imaging when the potential detriment of radiation exposure is considered. When CT-DSA is used in patients for whom radiation exposure is a concern, physicians could consider skipping the early arterial or venous phases to reduce exposure.

\section{CONCLUSIONS}

Dual-energy CT-DSA correlates well with conventional DSA in assessing the vascularity of spinal metastasis. It may serve as a potentially useful noninvasive tool for evaluating the vascularity of spinal metastasis.

\section{REFERENCES}

1. Mut M, Schiff D, Shaffrey ME. Metastasis to nervous system: spinal epidural and intramedullary metastases. J Neurooncol 2005;75: 43-56 CrossRef Medline

2. Gilbert RW, Kim JH, Posner JB. Epidural spinal cord compression from metastatic tumor: diagnosis and treatment. Ann Neurol 1978; 3:40-51 CrossRef Medline

3. de Ruiter GC, Nogarede CO, Wolfs JF, et al. Quality of life after different surgical procedures for the treatment of spinal metastases: results of a single-center prospective case series. Neurosurg Focus 2017;42:E17 CrossRef Medline

4. Robial N, Charles YP, Bogorin I, et al. Is preoperative embolization a prerequisite for spinal metastases surgical management? Orthop Traumatol Surg Res 2012;98:536-42 CrossRef Medline

5. Pazionis TJ, Papanastassiou ID, Maybody M, et al. Embolization of hypervascular bone metastases reduces intraoperative blood loss: a case-control study. Clin Orthop Relat Res 2014;472: 3179-87 CrossRef Medline

6. Kickuth $\mathrm{R}$, Waldherr $\mathrm{C}$, Hoppe $\mathrm{H}$, et al. Interventional management of hypervascular osseous metastasis: role of embolotherapy before orthopedic tumor resection and bone stabilization. AJR Am J Roentgenol 2008;191:W240-47 CrossRef Medline

7. McDonald K, Murphy LE, Eames N. Is it necessary to embolise all spinal metastases from primary renal tumours? J Orthop 2016;13: 472-74 CrossRef Medline

8. Qiao Z, Jia N, He Q. Does preoperative transarterial embolization decrease blood loss during spine tumor surgery? Interv Neuroradiol 2015;21:129-35 CrossRef Medline

9. Mendel E, Bourekas E, Gerszten P, et al. Percutaneous techniques in the treatment of spine tumors: what are the diagnostic and therapeutic indications and outcomes? Spine 2009;34:S93-100 CrossRef Medline

10. Schmidt R, Rupp-Heim G, Dammann F, et al. Surgical therapy of vertebral metastases: are there predictive parameters for intraoperative excessive blood loss despite preoperative embolization? $\mathrm{Tu}$ mori 2011;97:66-73 CrossRef Medline

11. Mauch JT, Carr CM, Cloft H, et al. Review of the imaging features of benign osteoporotic and malignant vertebral compression fractures. AJNR Am J Neuroradiol 2018;39:1584-92 CrossRef Medline

12. Jeong D, Bui M, Peterson D, et al. FDG avid breast cancer bone metastases silent on CT and scintigraphy: a case report with radiologic- 
pathologic correlation. Acta Radiol Open 2017;6:2058460117734243 CrossRef Medline

13. Al-Muqbel KM. Bone marrow metastasis is an early stage of bone metastasis in breast cancer detected clinically by F18-FDG-PET/CT imaging. Biomed Res Int 2017;2017:9852632 CrossRef Medline

14. Gondim Teixeira PA, Hossu G, Lecocq S, et al. Bone marrow edema pattern identification in patients with lytic bone lesions using digital subtraction angiography-like bone subtraction on large-area detector computed tomography. Invest Radiol 2014;49:156-64 CrossRef Medline

15. Gondim Teixeira PA, Gervaise A, Louis M, et al. Musculoskeletal wide detector CT: principles, techniques and applications in clinical practice and research. Eur J Radiol 2015;84:892-900 CrossRef Medline

16. Nishii $\mathrm{T}$, Kono AK, Nishio M, et al. Bone-subtracted spinal CT angiography using nonrigid registration for better visualization of arterial feeders in spinal arteriovenous fistulas. AJNR Am J Neuroradiol 2015;36:2400-06 CrossRef Medline

17. Yu L, Christner JA, Leng S, et al. Virtual monochromatic imaging in dual-source dual-energy CT: radiation dose and image quality. Med Phys 2011;38:6371-79 CrossRef Medline

18. Hayton A, Wallace A, Marks P, et al. Australian diagnostic reference levels for multi detector computed tomography. Australas Phys Eng Sci Med 2013;36:19-26 CrossRef Medline

19. Directorate-General for Energy, Directorate D, Nuclear Safety and Fuel Cycle, Unit D3, Radiation Protection. RADIATION PROTECTION No. 180 Diagnostic Reference Levels in Thirty-six European Countries (Part 2). European Commission; 2014. https://ec.europa.eu/energy/sites/ener/ files/documents/RP180\%20part2.pdf. Accessed January 15, 2018

20. Takase K, Sawamura Y, Igarashi K, et al. Demonstration of the artery of Adamkiewicz at multi-detector row helical CT. Radiology 2002; 223:39-45 CrossRef Medline

21. Kudo K, Terae S, Asano T, et al. Anterior spinal artery and artery of Adamkiewicz detected by using multi-detector row CT. AJNR Am J Neuroradiol 2003;24:13-17 Medline

22. Yoshioka K, Tanaka R, Takagi $\mathrm{H}$, et al. Ultra-high-resolution CT angiography of the artery of Adamkiewicz: a feasibility study. Neuroradiology 2018;60:109-15 CrossRef Medline

23. Mazura JC, Karimi S, Pauliah M, et al. Dynamic contrast-enhanced magnetic resonance perfusion compared with digital subtraction angiography for the evaluation of extradural spinal metastases: a pilot study. Spine 2014;39:E950-54 CrossRef Medline

24. Shi HB, Suh DC, Lee HK, et al. Preoperative transarterial embolization of spinal tumor: embolization techniques and results. AJNR Am J Neuroradiol 1999;20:2009-15 Medline

25. Suzuki H, Kondo T, Kuwatsuru R, et al. Decompressive surgery in combination with preoperative transcatheter arterial embolization: successful improvement of ambulatory function in renal cell carcinoma patients with metastatic extradural spinal cord compression. Int J Urol 2011;18:718-22 CrossRef Medline

26. Tomita K, Kawahara N, Kobayashi T, et al. Surgical strategy for spinal metastases. Spine 2001;26:298-306 CrossRef Medline
27. Tokuhashi $Y$, Matsuzaki $H$, Oda $H$, et al. A revised scoring system for preoperative evaluation of metastatic spine tumor prognosis. Spine 2005;30:2186-91 CrossRef Medline

28. Cappuccio M, Gasbarrini A, Van Urk P, et al. Spinal metastasis: a retrospective study validating the treatment algorithm. Eur Rev Med Pharmacol Sci 2008;12:155-60 Medline

29. Saha A, Peck KK, Lis E, et al. Magnetic resonance perfusion characteristics of hypervascular renal and hypovascular prostate spinal metastases: clinical utilities and implications. Spine 2014;39: E1433-40 CrossRef Medline

30. Clausen C, Dahl B, Christiansen Frevert S, et al. Inter-and intra-rater agreement in the assessment of the vascularity of spinal metastases using digital subtraction angiography tumor blush. Acta Radiol 2017;58:734-39 CrossRef Medline

31. Khadem NR, Karimi S, Peck KK, et al. Characterizing hypervascular and hypovascular metastases and normal bone marrow of the spine using dynamic contrast-enhanced MR imaging. AJNR Am J Neuroradiol 2012;33:2178-85 CrossRef Medline

32. Shimoyama S, Nishii T, Watanabe Y, et al. Advantages of 70-kV CT angiography for the visualization of the Adamkiewicz artery: comparison with 120-kV imaging. AJNR Am J Neuroradiol 2017;38: 2399-405 CrossRef Medline

33. Mendonca PR, Lamb P, Sahani DV. A flexible method for multimaterial decomposition of dual-energy CT images. IEEE Trans Med Imaging 2014;33:99-116 CrossRef Medline

34. Dinkel J, Khalilzadeh O, Phan CM, et al. Technical limitations of dual-energy CT in neuroradiology: 30-month institutional experience and review of literature. J Neurointerv Surg 2015;7:596602 CrossRef Medline

35. Gupta R, Phan CM, Leidecker C, et al. Evaluation of dual-energy CT for differentiating intracerebral hemorrhage from iodinated contrast material staining. Radiology 2010;257:205-11 CrossRef Medline

36. Kuno $\mathrm{H}$, Onaya $\mathrm{H}$, Iwata $\mathrm{R}$, et al. Evaluation of cartilage invasion by laryngeal and hypopharyngeal squamous cell carcinoma with dualenergy CT. Radiology 2012;265:488-96 CrossRef Medline

37. Hiyama T, Kuno H, Sekiya K, et al. Bone subtraction iodine imaging using area detector CT for evaluation of skull base invasion by nasopharyngeal carcinoma. AJNR Am J Neuroradiol 2019;40:135-41 CrossRef Medline

38. Ali IT, Wong WD, Liang T, et al. Clinical utility of dual-energy CT analysis of bone marrow edema in acute wrist fractures. $A J R A m J$ Roentgenol 2018;210:842-47 CrossRef Medline

39. Mallinson PI, Coupal TM, McLaughlin PD, et al. Dual-energy CT for the musculoskeletal system. Radiology 2016;281:690-707 CrossRef Medline

40. Yushkevich PA, Piven J, Hazlett HC, et al. User-guided 3D active contour segmentation of anatomical structures: significantly improved efficiency and reliability. Neuroimage 2006;31:1116-28 CrossRef Medline 